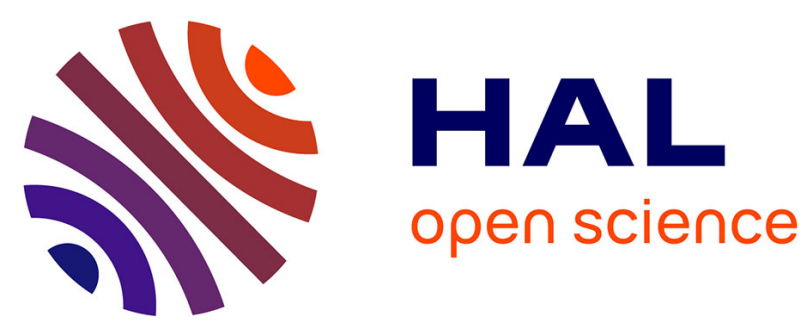

\title{
Structure and Biosynthesis of Xenoamicins from Entomopathogenic Xenorhabdus
}

\author{
Qiuqin Zhou, Florian Grundmann, Marcel Kaiser, Matthias Schiell, Sophie \\ Gaudriault, Andreas Batzer, Michael Kurz, Helge B. Bode
}

\section{- To cite this version:}

Qiuqin Zhou, Florian Grundmann, Marcel Kaiser, Matthias Schiell, Sophie Gaudriault, et al.. Structure and Biosynthesis of Xenoamicins from Entomopathogenic Xenorhabdus. Chemistry - A European Journal, 2013, 19 (49), pp.16772-16779. 10.1002/chem.201302481 . hal-01837255

\section{HAL Id: hal-01837255 \\ https://hal.science/hal-01837255}

Submitted on 29 May 2020

HAL is a multi-disciplinary open access archive for the deposit and dissemination of scientific research documents, whether they are published or not. The documents may come from teaching and research institutions in France or abroad, or from public or private research centers.
L'archive ouverte pluridisciplinaire HAL, est destinée au dépôt et à la diffusion de documents scientifiques de niveau recherche, publiés ou non, émanant des établissements d'enseignement et de recherche français ou étrangers, des laboratoires publics ou privés. 


\title{
Structure and Biosynthesis of Xenoamicins from Entomopathogenic Xenorhabdus
}

\author{
Qiuqin Zhou, ${ }^{[\mathrm{a}]}$ Florian Grundmann,${ }^{[\mathrm{a}]}$ Marcel Kaiser, ${ }^{[\mathrm{b}]}$ Matthias Schiell, ${ }^{[\mathrm{c}]}$ \\ Sophie Gaudriault, ${ }^{[d]}$ Andreas Batzer,${ }^{[c]}$ Michael Kurz, ${ }^{\left[{ }^{[c]}\right.}$ and Helge B. Bode ${ }^{*[a]}$
}

\begin{abstract}
During the search for novel natural products from entomopathogenic Xenorhabdus doucetiae DSM17909 and $X$. mauleonii DSM17908 novel peptides named xenoamicins were identified in addition to the already known antibiotics xenocoumacin and xenorhabdin. Xenoamicins are acylated tridecadepsipeptides consisting of mainly hydrophobic amino acids. The main derivative xenoamicin A (1) was isolated from X. mauleonii DSM17908, and its structure elucidated by detailed $1 \mathrm{D}$
\end{abstract}

and 2D NMR experiments. Detailed MS experiments, also in combination with labeling experiments, confirmed the determined structure and allowed structure elucidation of additional derivatives. Moreover, the xenoamicin biosynthesis gene cluster was identified and analyzed in $X$. doucetiae

Keywords: biosynthesis • natural products $\cdot$ peptides $\cdot$ structure elucidation
DSM17909, and its participation in xenoamicin biosynthesis was confirmed by mutagenesis. Advanced Marfey's analysis of $\mathbf{1}$ showed that the absolute configuration of the amino acids is in agreement with the predicted stereochemistry deduced from the nonribosomal peptide synthetase XabABCD. Biological testing revealed activity of 1 against Plasmodium falciparum and other neglected tropical diseases but no antibacterial activity.

\section{Introduction}

Natural products have always been a great source of novel bioactive compounds, in particular in the field of anti-infectives. As the number of multiresistant pathogens is increasing, it is necessary to continue research in the field of natural products, ${ }^{[1]}$ and thus novel approaches for the identification of natural products from well-established or novel natural-product producers are required. Additionally, novel molecular approaches also allow the activation of previously silent gene clusters or heterologous expression. ${ }^{[2,3]}$ Among novel natural-product producers, bacteria of the genus Xen-

[a] Q. Zhou, ${ }^{+}$F. Grundmann,$^{+}$Prof. Dr. H. B. Bode

Goethe-Universität Frankfurt

Department of Molecular Biotechnology

Max-von-Laue-Strasse 9, 60438 Frankfurt am Main (Germany)

E-mail: h.bode@bio.uni-frankfurt.de

[b] M. Kaiser

Swiss Tropical and Public Health Institute, Parasite Chemotherapy Socinstrasse 57, P.O. Box,

4002 Basel (Switzerland)

[c] M. Schiell, Dr. A. Batzer, Dr. M. Kurz

Sanofi R\&D, Industriepark Höchst 65926 Frankfurt am Main (Germany)

[d] Dr. S. Gaudriault

INRA, UMR 1333 Laboratoire DGIMI, 34095 Montpellier (France) and

Université Montpellier 2, UMR 1333 Laboratoire DGIMI 34095 Montpellier (France)

$\left.{ }^{+}\right]$These authors contributed equally to this work.

Supporting information for this article is available on the WWW under http://dx.doi.org/10.1002/chem.201302481. orhabdus have shown promise. ${ }^{[4-6]}$ The bacteria live in symbiosis with nematodes of the genus Steinernema and are released from the nematode gut when the nematode infects an insect. Besides insecticidal protein toxins Xenorhabdus also produces small molecules that are involved in insect virulence or act as antibiotics. Examples are the benzylideneacetone,${ }^{[7]}$ rhabduscin,,${ }^{[8]}$ xenocoumacins, ${ }^{[9]}$ xenorhabdins, ${ }^{[10]}$ xenofuranones ${ }^{[11]}$ nematophin, ${ }^{[12]}$ PAX-Peptides ${ }^{[13]}$ GameXPeptides $^{[14]}$ and even depsipeptides, such as xenematides, ${ }^{[15]}$ szentiamides, ${ }^{[15]}$ and xentrival peptides, ${ }^{[16]}$ which show antibiotic, antifungal, or cytotoxic activity. As several of the compounds may be released to protect the insect cadaver from food competitors living in the soil, they may also be potential anti-infectives. Here we describe the structure and biosynthesis of a novel class of large hydrophobic depsipeptides named xenoamicins from Xenorhabdus showing activity against Plasmodium and Trypanosoma.

\section{Results and Discussion}

During our screening for novel natural products by UPLCESI-MS of XAD-16 extracts ${ }^{[17]}$ from Xenorhabdus cultures in standard LB medium we identified a new family of natural products. According to characteristic neutral losses for amino acids such as valine, leucine, and isoleucine in their $\mathrm{MS}^{2}$ fragmentation patterns, these compounds were identified as peptides. ${ }^{[18]}$ Their molecular formulas were determined on the basis of MALDI-Orbitrap-MS data (Table S1 of the Supporting Information) revealing $\mathrm{C}_{64} \mathrm{H}_{109} \mathrm{~N}_{13} \mathrm{O}_{15}$ for xenoamicin A $\left(\mathbf{1} ; m / z, 1300.822[M+\mathrm{H}]^{+}, \Delta\right.$ ppm 0.5$)$ as the 
main product in $X$. mauleonii DSM17908. The determination of the molecular formula was facilitated and confirmed by the identification of the correct number of carbon and nitrogen atoms from labeling experiments with strain DSM 17908 in standard growth medium, ${ }^{13} \mathrm{C}$-labeled medium, and ${ }^{15} \mathrm{~N}$-labeled medium (Figure S1 of the Supporting Information), as described previously. ${ }^{[14,16]}$ Compound $\mathbf{1}$ (29.2 mg) was subsequently isolated from strain DSM17908 by preparative chromatography with HPLC-UV/ELSD in a three step protocol (Figures S2-S4 of the Supporting Information). The first two chromatographic steps were performed on $\mathrm{C}_{18}$ columns with different particle sizes under acidic conditions. In the third step the selectivity was changed through a higher $\mathrm{pH}$ value of the eluent. Due to the lack of a chromophore, $\mathbf{1}$ shows only weak absorption in the UV spectrum even at $215 \mathrm{~nm}$. Despite several peaks with a strong absorption at $215 \mathrm{~nm}$ in the extract of $X$. mauleonii DSM17908, 1 could easily be identified with the universal signal of the evaporative light scattering detector (ELSD). Due to the relatively large amount of $\mathbf{1}$ in the extract of DSM17908 a large signal in the ELSD but not in the UV spectra could be observed, whereas other compounds in this extract with a chromophore gave large signals in both spectra (Figure S2 of the Supporting Information). Thus, the ELSD signal gives a very good indication of the actual amount of the individual compounds in the extract, independent of the physicochemical properties, and therefore ELSD is especially valuable for the detection of peptides without any chromophore. $^{[19]}$

The structure of $\mathbf{1}$ was determined by comprehensive NMR studies. In most standard solvents, such as $\left[\mathrm{D}_{6}\right] \mathrm{DMSO}, \mathrm{CD}_{3} \mathrm{OD}$, or $\mathrm{CDCl}_{3}, \mathrm{NMR}$ spectra of poor quality were obtained. The resonances of the amide protons in particular appeared as very broad signals (Figures S5, S6 and Annex 1 of the Supporting Information). However, in $\left[\mathrm{D}_{6}\right]$ benzene at $293 \mathrm{~K}$ well-resolved NMR spectra with reasonable dispersion of the amide signals were obtained (Figure S6 of the Supporting Information). The assignment of all proton and carbon resonances was carried out with various 2D NMR techniques, including DQF-COSY, TOCSY, ROESY, multiplicity-edited HSQC, HSQC-TOCSY, and HMBC spectra (Table 1, Annexes 1-20 in the Supporting Information). The assignment was hampered by the presence of two sets of signals in a ratio of 1:1 resulting from proline cis/trans conformers (see below). Primary analysis of the spin systems in the TOCSY and HSQC-TOCSY spectra revealed the presence of 13 amino acid residues: five valines, one leucine, one isoleucine, two alanines, two prolines, one threonine, and one $\beta$-alanine residue. In addition a butyric acid moiety was observed. The two sets of signals are caused by different orientations of the amide bond between the $\beta$ alanine residue and one of the proline residues. In the cis conformer an intensive ROE correlation between proline $\mathrm{H} \alpha$ and $\beta$-alanine $\mathrm{H} \beta$ is observed. In the the trans conformer such an intensive ROE should appear between proline $\mathrm{H} \delta$ and $\beta$-alanine $\mathrm{H} \beta$. Unfortunately, this ROE cannot be assigned, because of peak overlaps between proline $\mathrm{H} \delta$ and the $\mathrm{H} \beta$ of $\beta$-alanine. The presence of a cis and a trans conformer is also confirmed by the difference in ${ }^{13} \mathrm{C}$ chemical shifts of $C \beta$ and $C \gamma$ of proline $\left[\Delta(\beta \gamma)=\delta\left({ }^{13} C \beta-{ }^{13} C \gamma\right)\right] \cdot{ }^{[20]}$ The differences for the cis and trans conformers are $\Delta(\beta \gamma)=$ $8.83 \mathrm{ppm}$ and $\Delta(\beta \gamma)=5.17 \mathrm{ppm}$, respectively, which are in agreement with literature data. ${ }^{[20]}$ Due to overlap of the carbon resonances in the carbonyl region and line broadening of some amide protons, the HMBC spectrum was not sufficient for the sequential assignment (see Annex 3 of the Supporting Information). Therefore, the peptide sequence was determined by analysis of ROE correlations which resulted in a depsipeptide structure with ring formation between the C-terminal valine residue and the threonine side chain at position 7 (Figure 1). The ring closure could be confirmed by an ROE between threonine $\mathrm{H} \beta$ and valine $14 \mathrm{H} \alpha$ in both conformers, whereby the ROE of the cis conformer is only weak (Annex 13 of the Supporting Information). All other sequential NH/H $\alpha$ ROE correlations of both conformers were observed with the exception of the correlation between valine 10 and alanine 11 in both conformers. Nevertheless, the connection of valine 10 and alanine 11 was in accordance with the molecular formula and was shown by MS fragmentation data (see below). The assignment of the single spin systems to the corresponding conformer was confirmed by additional long-range ROE correlations between valine $10 \mathrm{NH}$ and isoleucine $6 \mathrm{H} \alpha$. Moreover, a long-range ROE correlation between threonine $\mathrm{H} \beta$ and alanine $11 \mathrm{H} \alpha$ confirmed the assignment of the trans conformer. The N-terminal proline residue is acylated with butyric acid, as could be concluded from an ROE correlation between $\mathrm{H} \alpha$ of the acid and $\mathrm{H} \alpha$ of proline in position 2. Unfortunately, the alanine $\mathrm{NH}$ in position 11 of the trans conformer could not be detected due to very broad signals.

The configuration of the amino acids in $\mathbf{1}$ was elucidated by using the advanced Marfey's method ${ }^{[16,21,22]}$ leading to the identification of the L configuration for isoleucine and both proline residues. The D configuration was revealed for leucine, allo-threonine, and both alanine residues. The ratio of $\mathrm{L}$ to $\mathrm{D}$ valine was found to be 1:4. Therefore, four valine residues in $\mathbf{1}$ may have the D configuration and one valine residue has the L configuration (Figure 2, Table S2 of the Supporting Information). However, the stereochemical assignment of the valine residues was not possible at this stage.

To verify and support the structure elucidation by NMR spectroscopy and to enable the structural elucidation of further derivatives in $X$. doucetiae DSM17909, MS-based structure elucidation was additionally performed for $\mathbf{1}$ in DSM1908. To confirm the building blocks, standard ${ }^{12} \mathrm{C}-\mathrm{L}-$ amino acids were added to bacterial cultures growing in a reversed labeling experiment in fully ${ }^{13} \mathrm{C}$ labeled medium (Figure S7 of the Supporting Information), as described previously. ${ }^{[14,16]}$ Incorporation could be detected by HPLC-ESIMS as shifts to lower masses of $\mathrm{m} / z 1.5$ for $\beta$-alanine, 2 for threonine, 2.5 for proline, 3 for isoleucine, 3 for leucine, 1.5 for alanine, and 2.5 for valine, related to half of the numbers of carbon atoms incorporated due to the presence of doubly charged ions. 
Table 1. NMR spectroscopic data of conformer mixture $\left(600 \mathrm{MHz}\left({ }^{1} \mathrm{H}\right)\right.$, $150 \mathrm{MHz}\left({ }^{13} \mathrm{C}\right)$ in $\left[\mathrm{D}_{6}\right]$ benzene) of xenoamicin A (1), $\delta$ in ppm. Spin system numbering from acylated $\mathrm{N}$ - to C-terminus. Only key sequential ROESY correlations are listed. Pos. $=$ Position

\begin{tabular}{|c|c|c|c|c|c|c|c|}
\hline \multirow[t]{2}{*}{ Amino acid } & \multirow[t]{2}{*}{ Pos. } & \multicolumn{3}{|c|}{ trans Conformer } & \multicolumn{3}{|c|}{ cis Conformer } \\
\hline & & $\delta_{\mathrm{C}}$ & $\delta_{\mathrm{H}}$ & ROESY & $\delta_{\mathrm{C}}$ & $\delta_{\mathrm{H}}$ & ROESY \\
\hline \multirow[t]{5}{*}{ 1-BA } & $\alpha$ & 36.46 & 2.34 & $2-\alpha$ & 36.46 & 2.34 & $2-\alpha$ \\
\hline & $\alpha$ & 36.46 & 1.95 & & 36.46 & 1.95 & \\
\hline & $\beta$ & 18.63 & 1.90 & & 18.63 & 1.90 & \\
\hline & $\beta$ & & 1.85 & & & 1.85 & \\
\hline & $\gamma$ & 14.23 & 0.98 & & 14.23 & 0.98 & \\
\hline \multirow[t]{7}{*}{ 2-Pro } & $\alpha$ & 60.30 & 4.58 & $1-\alpha, 3-\mathrm{NH}$ & 60.30 & 4.58 & $1-\alpha, 3-\mathrm{NH}$ \\
\hline & $\beta$ & 29.54 & 1.82 & $3-\mathrm{NH}$ & 29.54 & 1.82 & $3-\mathrm{NH}$ \\
\hline & $\beta$ & & 1.47 & $3-\mathrm{NH}$ & & 1.47 & $3-\mathrm{NH}$ \\
\hline & $\gamma$ & 24.96 & 2.08 & & 24.96 & 2.08 & \\
\hline & $\gamma$ & & 1.29 & & & 1.29 & \\
\hline & $\delta$ & 47.13 & 3.28 & & 47.13 & 3.28 & \\
\hline & $\delta$ & & 2.73 & & & 2.73 & \\
\hline \multirow[t]{3}{*}{$3-\mathrm{Ala}^{[\mathrm{a}]}$} & $\mathrm{NH}$ & & 8.71 & $2-\alpha, 2-\beta$ & & 8.71 & $2-\alpha, 2-\beta$ \\
\hline & $\alpha$ & $48.06^{[\mathrm{b}]}$ & 5.01 & $4-\mathrm{NH}$ & $48.01^{[b]}$ & 5.03 & 4-NH \\
\hline & $\beta$ & 16.56 & 1.69 & & 16.56 & 1.67 & \\
\hline \multirow[t]{5}{*}{$4-\mathrm{Val}^{[\mathrm{a}]}$} & $\mathrm{NH}$ & & 7.82 & $3-\alpha$ & & 7.82 & $3-\alpha$ \\
\hline & $\alpha$ & 60.04 & 4.84 & $5-\mathrm{NH}$ & 60.08 & 4.78 & $5-\mathrm{NH}$ \\
\hline & $\beta$ & 30.70 & 2.25 & & 30.70 & 2.25 & \\
\hline & $\gamma$ & 19.63 & 1.10 & & 19.64 & 1.06 & \\
\hline & $\gamma$ & 19.45 & 1.04 & & 19.38 & 1.00 & \\
\hline \multirow[t]{6}{*}{ 5-Leu } & $\mathrm{NH}$ & & 9.22 & $4-\alpha$ & & 9.23 & $4-\alpha$ \\
\hline & $\alpha$ & 50.76 & 5.14 & 6-NH & 50.93 & 5.13 & 6-NH \\
\hline & $\beta$ & 40.34 & 2.03 & & 40.34 & 2.00 & \\
\hline & $\gamma$ & $25.56^{[\mathrm{b}]}$ & 1.97 & & $25.50^{[b]}$ & 1.97 & \\
\hline & $\delta$ & 22.59 & 1.09 & & 22.59 & 1.09 & \\
\hline & $\delta$ & 23.45 & 1.10 & & 23.45 & 1.10 & \\
\hline \multirow[t]{7}{*}{ 6-Ile } & NH & & 8.70 & $5-\alpha$ & & 8.74 & $5-\alpha$ \\
\hline & $\alpha$ & 58.05 & 5.00 & $\begin{array}{l}7-\mathrm{NH}, 7-\beta, \\
10-\mathrm{NH}\end{array}$ & 58.01 & 4.92 & $\begin{array}{l}\text { 7-NH, 7- } \beta, \\
10-\mathrm{NH}\end{array}$ \\
\hline & $\beta$ & 34.91 & 2.34 & & 34.83 & 2.34 & \\
\hline & $\gamma 1$ & 9.75 & 0.96 & & 9.75 & 0.94 & \\
\hline & $\gamma^{2}$ & 25.44 & 1.72 & & 25.39 & 1.72 & \\
\hline & $\gamma^{2}$ & & 1.52 & & & 1.52 & \\
\hline & $\delta$ & 15.91 & 1.14 & & 15.87 & 1.12 & \\
\hline \multirow[t]{4}{*}{ 7-Thr } & $\mathrm{NH}$ & & 9.60 & $6-\alpha$ & & 9.54 & $6-\alpha$ \\
\hline & $\alpha$ & 56.71 & 5.18 & 8-NH & 56.14 & 5.15 & 8-NH \\
\hline & $\beta$ & 70.03 & 6.05 & $\begin{array}{l}6-\alpha, 11-\alpha \\
14-\alpha\end{array}$ & 71.82 & 5.82 & $6-\alpha, 14-\alpha$ \\
\hline & $\gamma$ & 17.66 & 1.47 & & 17.24 & 1.49 & \\
\hline \multirow[t]{5}{*}{ 8-Val } & $\mathrm{NH}$ & & 9.03 & $7-\alpha, 7-\beta$ & & 8.84 & $7-\alpha, 7-\beta$ \\
\hline & $\alpha$ & 61.88 & 4.62 & 9-NH & 61.54 & 4.56 & 9-NH \\
\hline & $\beta$ & 29.36 & 2.57 & & 29.54 & 2.56 & \\
\hline & $\gamma$ & 19.72 & 1.37 & & 19.71 & 1.33 & \\
\hline & $\gamma$ & 19.55 & 1.27 & & 19.93 & 1.28 & \\
\hline \multirow[t]{5}{*}{ 9-Val } & NH & & 8.97 & $8-\alpha$ & & 8.85 & $8-\alpha$ \\
\hline & $\alpha$ & 60.82 & 4.76 & $10-\mathrm{NH}$ & 60.57 & 4.79 & $10-\mathrm{NH}$ \\
\hline & $\beta$ & $30.45^{[b]}$ & 2.55 & & $30.49^{[b]}$ & 2.56 & \\
\hline & $\gamma$ & 19.99 & 1.24 & & 19.91 & 1.25 & \\
\hline & $\gamma$ & 19.99 & 1.24 & & 19.91 & 1.25 & \\
\hline \multirow[t]{5}{*}{ 10-Val } & $\mathrm{NH}$ & & 9.15 & $6-\alpha, 9-\alpha$ & & 9.01 & $6-\alpha, 9-\alpha$ \\
\hline & $\alpha$ & 60.30 & 4.90 & & 58.64 & 5.49 & \\
\hline & $\beta$ & 29.68 & 2.56 & & 31.07 & 2.38 & \\
\hline & $\gamma$ & $19.44^{[b]}$ & 1.23 & & $19.50^{[b]}$ & 1.23 & \\
\hline & $\gamma$ & 19.36 & 1.18 & & 19.09 & 1.17 & \\
\hline 11-Ala & $\mathrm{NH}$ & & & & & 9.35 & \\
\hline & $\alpha$ & 49.93 & 5.45 & $7-\beta, 12-\mathrm{NH}$ & 50.31 & 5.16 & $12-\mathrm{NH}$ \\
\hline & $\beta$ & 17.24 & 1.75 & & 19.29 & 1.58 & \\
\hline $12-\beta-A l a$ & $\mathrm{NH}$ & & 7.97 & $11-\alpha$ & & 6.70 & $11-\alpha$ \\
\hline & $\alpha$ & 35.66 & 4.20 & & 35.79 & 4.42 & $13-\alpha$ \\
\hline & $\alpha$ & & 3.38 & & & 4.26 & \\
\hline & $\beta$ & 34.80 & 2.84 & & 35.13 & 3.00 & \\
\hline & $\beta$ & & 2.18 & & & 2.35 & $13-\alpha$ \\
\hline
\end{tabular}

Table 1. (Continued)

\begin{tabular}{llllllll}
\hline Amino acid & Pos. & \multicolumn{3}{c}{ trans Conformer } & \multicolumn{3}{c}{ cis Conformer } \\
& & $\delta_{\mathrm{C}}$ & $\delta_{\mathrm{H}}$ & ROESY & $\delta_{\mathrm{C}}$ & $\delta_{\mathrm{H}}$ & ROESY \\
\hline 13-Pro & $\alpha$ & 63.19 & 4.47 & & 61.47 & 4.26 & $12-\alpha, 12-\beta$ \\
& $\beta$ & 30.21 & 2.17 & & 31.39 & 1.76 & \\
& $\beta$ & & 1.73 & & & 1.66 & \\
& $\gamma$ & 25.04 & 1.40 & & 22.56 & 1.26 & \\
& $\gamma$ & & 1.68 & & & 1.11 & \\
& $\delta$ & 46.57 & 3.20 & $14-\mathrm{NH}$ & 46.48 & 3.54 & $14-\mathrm{NH}$ \\
& $\delta$ & & 2.81 & $14-\mathrm{NH}$ & & 3.22 & $14-\mathrm{NH}$ \\
& $\mathrm{NH}-\mathrm{Val}$ & & 6.60 & $7-\beta, 13-\delta$ & & 6.65 & $13-\delta$ \\
& $\alpha$ & 57.70 & 4.69 & $7-\beta$ & 56.14 & 4.51 & $7-\beta$ \\
& $\beta$ & 32.77 & 2.27 & & 32.05 & 2.17 & \\
& $\gamma$ & 18.83 & 0.97 & & 18.81 & 0.66 & \\
& $\gamma$ & 18.33 & 0.96 & & 16.98 & 0.63 & \\
& & & & & & & \\
\end{tabular}

[a] Interchangeable spin systems in the different conformers at the same position. [b] Interchangeable positions in the spin systems of the different conformers at the same position.

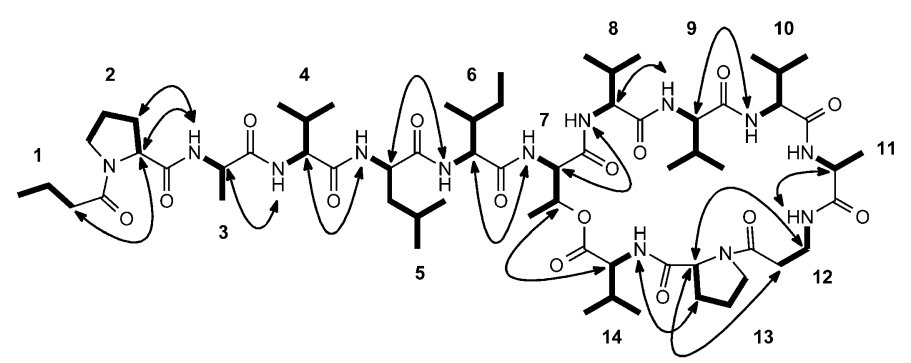

Figure 1. Connectivities in xenoamicin A (1), as determined by COSY and TOCSY (bold lines). Arrows indicate ROESY correlations used for the sequential assignment. Additionally the numeration of the spin systems is shown.

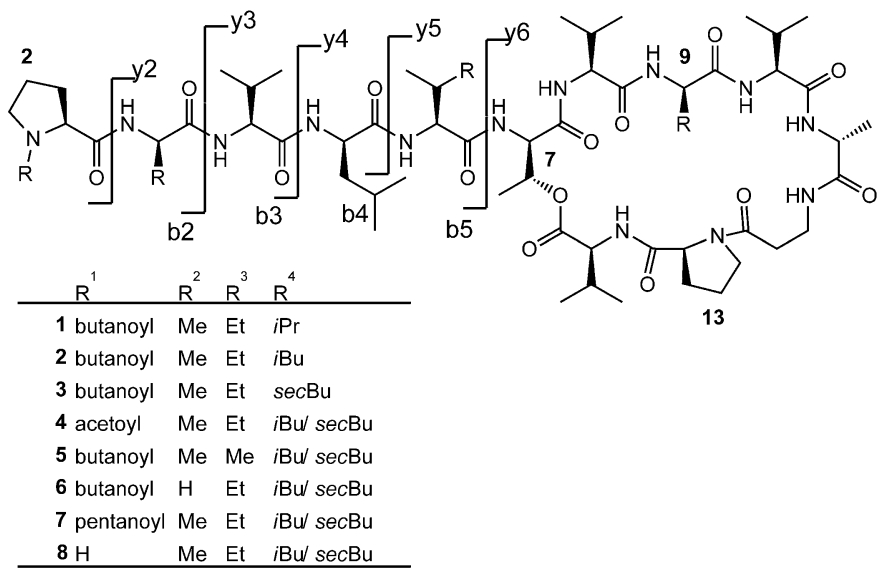

Figure 2. Structures of xenoamicins A-H (1-8) indicating b2-b5 and y2y5 fragmentation ions of the $\mathrm{MS}^{2}$ spectra. No differentiation between leucine or isoleucine at position $9\left(\mathrm{R}^{4}\right)$ could be obtained for 4-8.

The more intense doubly charged ions were used for fragmentation and showed mainly b2-b5 and y2-y6 ions (Figure 2, Figure S8 and Table S3 of the Supporting Information), as xenoamicins exhibited a characteristic collisioninduced dissociation (CID) $\mathrm{MS}^{2}$ fragmentation pattern. However, as the ring stayed intact in the CID experiment, the sequence of the ring could not be determined. There- 


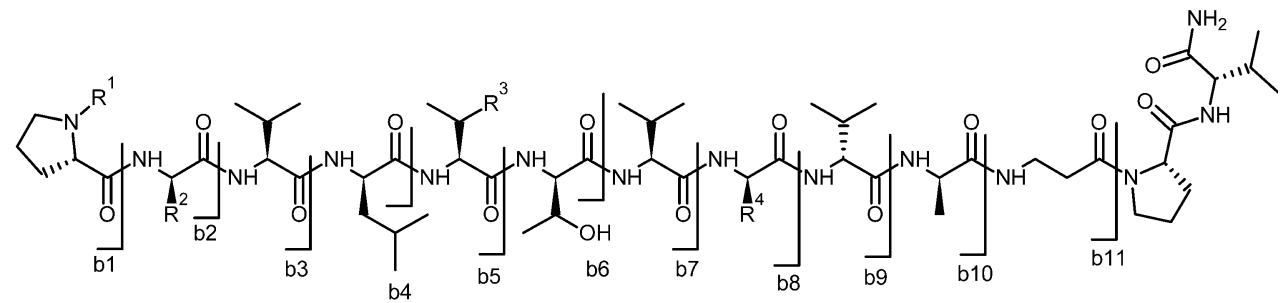

Figure 3. Structures of linear xenoamicins A-C (1-3) after reaction with $\mathrm{NH}_{3}$. Described b1-b11 ions could be observed in $\mathrm{MS}^{n}$ fragmentations. For $\mathrm{R}^{1}-$ $\mathrm{R}^{4}$ see Figure 2 .

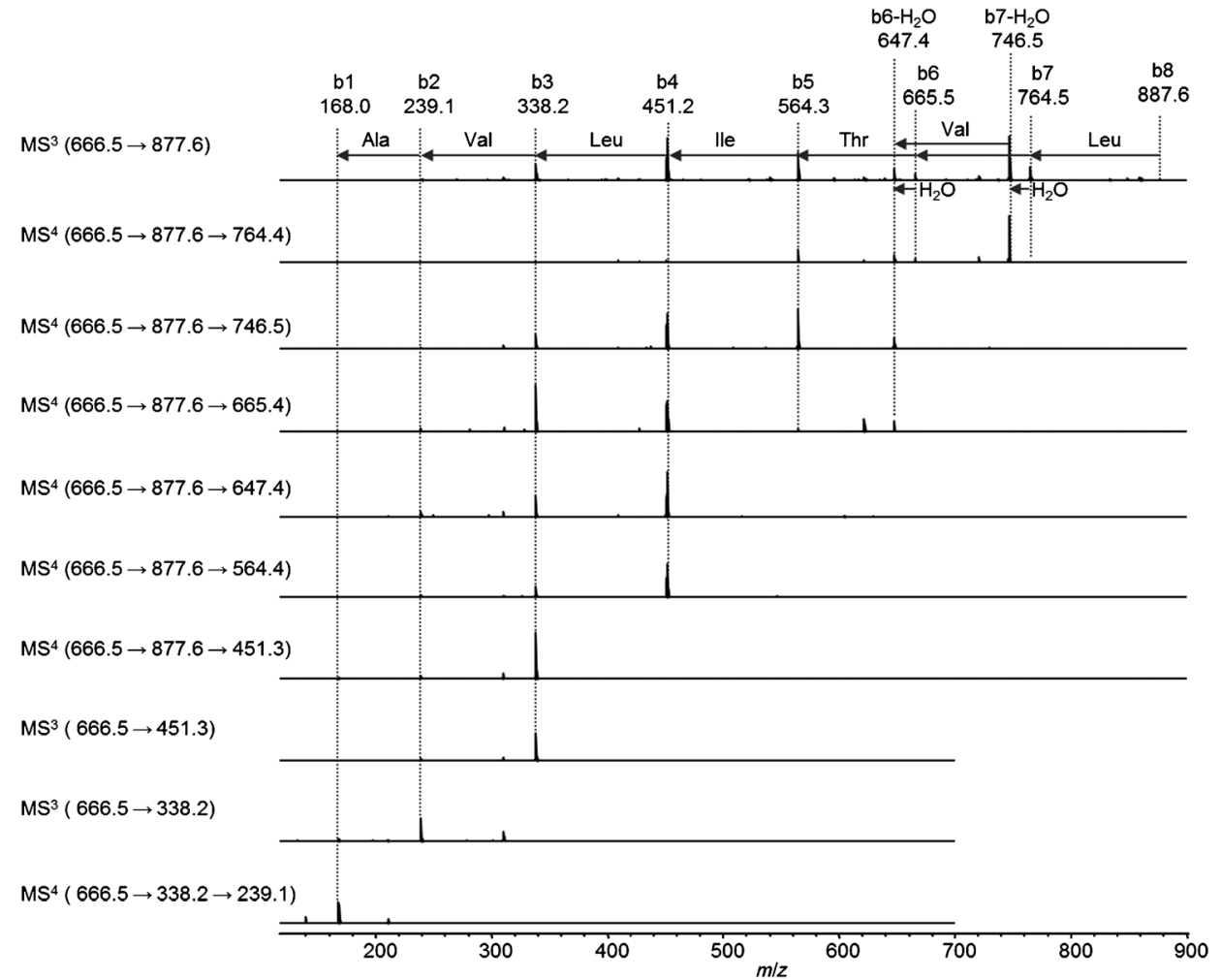

Figure 4. $\mathrm{MS}^{3}$ and $\mathrm{MS}^{4}$ spectra of linear xenoamicin B (2) after reaction with $\mathrm{NH}_{3}$. According to $\mathrm{MS}^{3}$ and $\mathrm{MS}^{4}$ spectra, b2-b5 and b8 ions could be assigned in $\mathrm{MS}^{2}$ spectra. Assignment of b9-b11 ions were proposed due to complex $\mathrm{MS}^{3}$ and $\mathrm{MS}^{4}$ fragmentation.

fore, xenoamicin-containing extracts were hydrolyzed with $28 \%$ aqueous $\mathrm{NH}_{3}$ solution, which resulted in positive mass shifts of $8.5 \mathrm{Da}$ and $9 \mathrm{Da}$ in doubly charged species for the addition of $\mathrm{NH}_{3}$ (17 Da; resulting in the linear amide) or for the addition of water (18 Da; resulting in the linear acid), respectively (Figure 3, Table S4 of the Supporting Information). ${ }^{[23]}$ Based on CID $\mathrm{MS}^{2}$ of the linearized peptide (Figure $\mathrm{S} 9$ of the Supporting Information) and $\mathrm{MS}^{n}$ experiments (Figure S10 of the Supporting Information), b1-b8 ions could be identified and also used for determination of the amino acid sequence of the ring, which confirmed the sequence of $\mathbf{1}$ as determined by NMR spectroscopy. The same analyses were performed for compounds $\mathbf{2}$ and $\mathbf{3}$, which appear in $X$. mauleonii DSM17908 and $X$. doucetiae DSM17909 (Figures S8 and S9, Tables S3 and S4 of the Sup- porting Information). Both show $\mathrm{m} / z 1314.8[M+\mathrm{H}]^{+}$indicating the presence of an additional methyl group in both compounds. The location of this additional methyl group in $\mathbf{2}$ and $\mathbf{3}$ could be identified as position 9 due to the difference between $\mathrm{b} 7$ and b8 ions (Figures 3 and 4; Figure S11 of the Supporting Information). In 1 the difference between the b7 and b8 ions of $99 \mathrm{Da}$ represents the neutral loss of a valine building block, whereas the difference in $\mathbf{2}$ and $\mathbf{3}$ of $113 \mathrm{Da}$ indicates leucine or isoleucine, respectively. Isobaric leucine and isoleucine could be distinguished by analyzing the results from inverse feeding experiments (Figure S7 of the Supporting Information). By feeding ${ }^{12} \mathrm{C}$-leucine to a culture in ${ }^{13} \mathrm{C}$-enriched media two shifts of $3 \mathrm{Da}$ from the double charged species could be seen for $\mathbf{2}$ and show the incorporation of two leucine residues instead of one leucine residue in $\mathbf{1}$ and $\mathbf{3}$. For $\mathbf{3}$ two shifts for isoleucine could be observed instead of one shift in $\mathbf{1}$ and $\mathbf{2}$ after the feeding of ${ }^{12} \mathrm{C}$-isoleucine. The extracts of the inverse feeding experiments were also hydrolyzed with $28 \%$ aqueous $\mathrm{NH}_{3}$ solution. However, the intensity of the b6 and b7 ions of the ${ }^{13} \mathrm{C}$-peptides with incorporated ${ }^{12} \mathrm{C}$-leucine or ${ }^{12} \mathrm{C}$-isoleucine was too low for detection (Table S4 of the Supporting Information). In summary, $\mathrm{MS}^{4}$ fragmentation and CID $\mathrm{MS}^{2}$ fragmentation of the linearized peptides together with inverse feeding experiments were sufficient for the complete sequential structural elucidation of $\mathbf{1}-\mathbf{3}$.

Additionally, xenoamicins D-H (4-8) could be detected in DSM17909, but not in DSM17908, as doubly charged species $\left([M+2 \mathrm{H}]^{2+}, m / z\right.$ 644.0, 651.0, 651.0, 665.0), although these were only produced in trace amounts (Table S1 of the 
Supporting Information). Their structure could only partially be determined from their b2-b5 and y2-y6 ions in $\mathrm{MS}^{2}$ spectra (Figure S12 and Table S5 of the Supporting Information). Determination of the ring amino acids of 4-8 was not possible. However, as variability in the ring was only observed at position 9 in compounds $\mathbf{1}-\mathbf{3}$, this may also be the variable position for compounds $\mathbf{4 - 8}$, although no differentiation between leucine and isoleucine is possible. As described above, the differentiation of leucine and isoleucine can only be achieved by reversed feeding experiments and subsequent linearization if the structure elucidation is performed by MS. However, the amounts of 4-8 were not sufficient for further characterization.

Nevertheless, 5 and $\mathbf{6}$ show variability in positions 6 and 3 , respectively. Furthermore, two additional N-terminal acyl moieties, acetate and pentanoate, were observed in $\mathbf{4}$ and $\mathbf{8}$. Experimentally proven characterization of the ring amino acids is not essential for elucidation of these variabilities.

The b2 ion of $\mathbf{4}$ with a mass of 211 Da revealed that acetate is acylated with the N-terminal proline residue. The neutral loss of proline is $97 \mathrm{Da}$ and that of alanine $71 \mathrm{Da}$. Therefore the remaining component of the b2 ions had a mass of $43 \mathrm{Da}$ indicating acetate. By comparison of the b2 ions with $211 \mathrm{Da}$ and the y2 ions with $1147 \mathrm{Da}$, alanine in position 3 is confirmed. Valine in position 4 , leucine in position 5 , and isoleucine in position 6 could be predicted by the mass shifts of 99, 113, and $113 \mathrm{Da}$ between the y3, y4, y5, and y6 ions, respectively. Leucine and isoleucine cannot be distinguished by their mass, but it can be assumed that leucine is incorporated at position 5 and isoleucine at position 6, as in 1-3. However, CID $\mathrm{MS}^{2}$ fragmentation was not sufficient for the analysis of the ring. Therefore, it had to be assumed that the ring consists of the same or very similar building blocks as in 1-3. However, because the y6 ions of 4 showed a mass of $751 \mathrm{Da}$, like for the y6 ions of $\mathbf{2}$ and $\mathbf{3}$, we propose that leucine or isoleucine was incorporated at position 9 , as in $\mathbf{2}$ and $\mathbf{3}$, and that all other amino acids in the ring were as in $\mathbf{1}-\mathbf{3}$.

The CID MS $^{2}$ fragmentation revealed that compound $\mathbf{5}$ has alanine in position 3 , valine in position 4 , leucine in position 5 , and valine in position 6 due to the shifts of 71, 99, 113 , and $99 \mathrm{Da}$ between the $\mathrm{y} 2, \mathrm{y} 3, \mathrm{y} 4, \mathrm{y} 5$, and y6 ions, respectively. Therefore this compound shows variability in position 6 due to the incorporation of valine instead of isoleucine. The b2 ions with a mass of $239 \mathrm{Da}$ revealed a butanoyl acid unit in position 1 . The obtained y6 ions for compound 5 exhibited the same size as for compound $\mathbf{4 , 6}, \mathbf{7}$, and $\mathbf{8}$, thus the structure shown in Figure 2 resulted.

In compound $\mathbf{6}$ glycine instead of alanine is incorporated in position 3, as revealed by the mass shift of $57 \mathrm{Da}$ between the $\mathrm{y} 2$ and $\mathrm{y} 3$ ions. According to the shifts between $\mathrm{y} 3, \mathrm{y} 4$, $\mathrm{y} 5$, and $\mathrm{y} 6$ ions, valine, leucine, and isoleucine are incorporated in positions 4,5 , and 6 , respectively. The loss of $14 \mathrm{Da}$ of the b2 ions in comparison with the b2 ions of $\mathbf{1}, \mathbf{2}, \mathbf{3}$, and 5 resulted from incorporation of glycine instead of alanine.

Compound $\mathbf{7}$ showed the same mass shifts as $\mathbf{2 - 4}$. Therefore, the same amino acid sequence can be assumed. How- ever, the b2 ions with a mass of 253 Da showed that 7 contained a pentanoyl moiety as the difference of the $y 2$ and the $\mathrm{y} 3$ ions indicated an alanine in position 3 . When the mass of alanine and proline were subtracted from the mass of the b2 ions the mass of a pentanoyl moiety is left.

The $\mathrm{MS}^{2}$ spectrum of $\mathbf{8}$ is more complex. Therefore the $\mathrm{MS}^{2}$ spectrum of the sodium adducts of $\mathbf{8}$ is additionally presented (Figure S12 of the Supporting Information). The y2y6 ions showed that $\mathbf{8}$ exhibited the same amino acid sequence and ring size as $\mathbf{2 - 4}$ and 7. Taking the molecular mass of $1244 \mathrm{Da}$ into account, it can be assumed that the $\mathrm{N}$ terminus of $\mathbf{8}$ is not acylated.

To fully assign the absolute configuration of xenoamicin, which was not completely possible from the NMR data and amino acid analysis, the biosynthesis gene cluster was identified and analyzed. As the genome of $X$. mauleonii DSM17908 is not available yet, the already finished genome sequence of strain DSM17909 (originally strain FRM16) (http://www.genoscope.cns.fr/agc/microscope/home/in-

dex.php) was searched for a biosynthesis gene cluster encoding nonribosomal peptide synthetases (NRPS) involved in xenoamicin biosynthesis $(x a b)$ by using the antismash software tool. ${ }^{[24-26]}$ The only candidate that fits the predicted biosynthesis gene cluster encodes five nonribosomal peptidases XabABCD and an aspartic acid decarboxylase XabE putatively involved in the formation of $\beta$-alanine. ${ }^{[14,27,28]}$

To prove that this gene cluster is indeed involved in xenoamicin biosynthesis, the gene $x a b B$ encoding the second NRPS was disrupted by plasmid insertion. Comparison between the $x a b B$ :cat mutant and the wild type showed complete loss of 1-8 in the mutant, whereas all other natural products such as such as xenorhabdins and xenocoumacins were still produced (Figures S13 and S14 of the Supporting Information).

Overall 13 modules and all domains for the biosynthesis of the xenoamicins could be identified (Figure 5, Table S6 of the Supporting Information). The presence of an adenylation (A) domain specific for $\beta$-alanine was readily detectable due to the differences in the specificity conferring code (Table S7 of the Supporting Information). ${ }^{[29-31]}$ Analysis of the condensation $(\mathrm{C})$ domains $^{[32]}$ revealed the presence of dual condensation/epimerization $(\mathrm{C} / \mathrm{E})$ domains ${ }^{[33]}$ in modules $3,5,7,9$, and 11 . Thus, it can be assumed that the amino acids incorporated in the previous modules $2,4,6,8$, and 10 are epimerized, which is in accordance with the results from the advanced Marfey's analysis (Table S2 of the Supporting Information). For example, exclusively L-proline and D-alanine were detected in the Marfey's analysis and only $\mathrm{C}$ and $\mathrm{C} / \mathrm{E}$ domains were identified following the modules responsible for the incorporation of proline (modules 1 and 12) and alanine (modules 2 and 10), respectively. Furthermore, 1-3 were identical in both strains according to the MS analysis of the linearized peptides as well as the retention times of cyclic or linear peptides. Thus, the absolute configuration of all amino acids in xenoamicins can be predicted accordingly and this also allows the stereochemical assignment for the valine moieties to be made (Figure 2). 


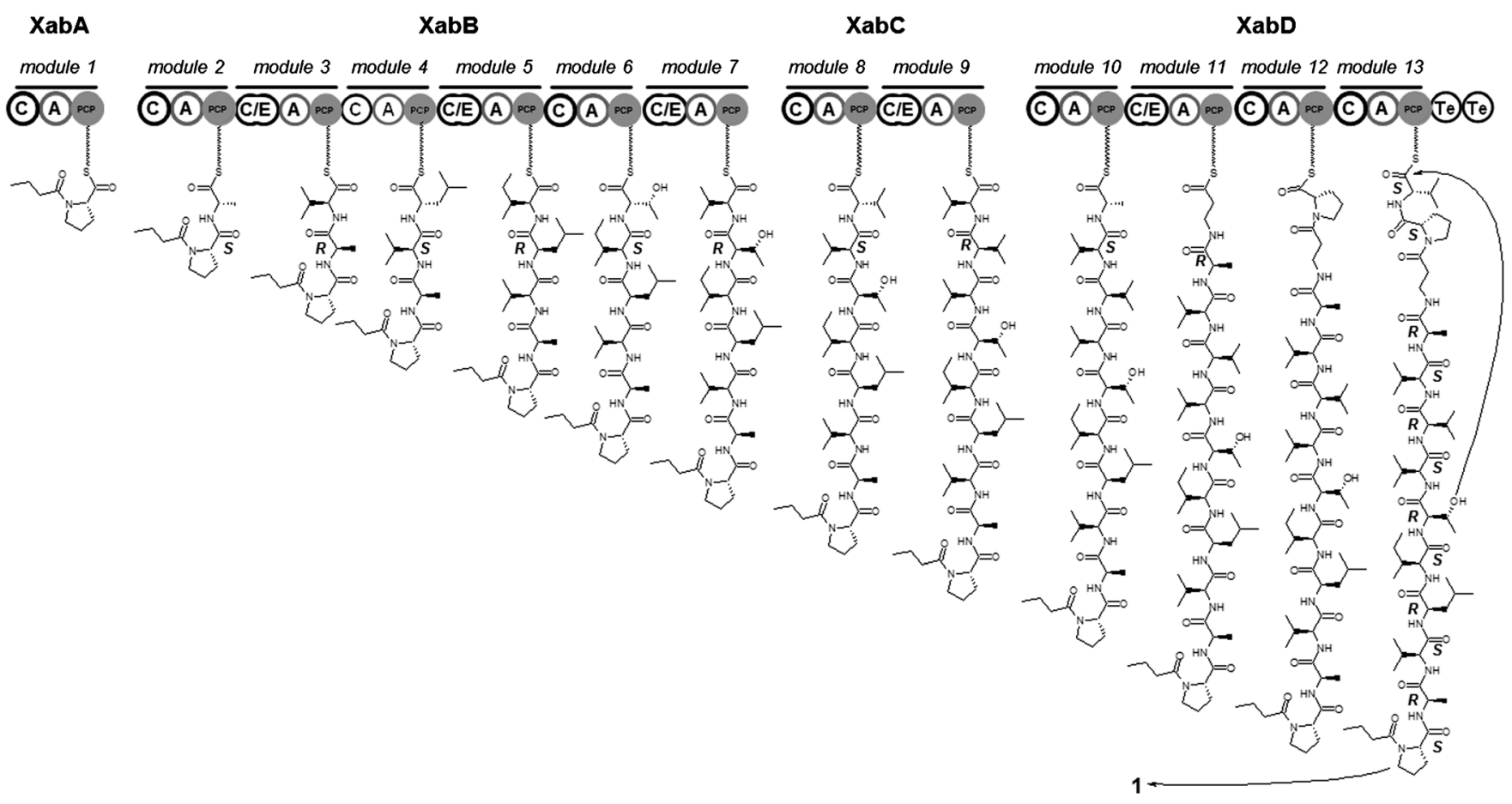

Figure 5. Proposed biosynthesis of xenoamicin A (1) in X. doucetiae DSM 17909, showing the nonribosomal peptide synthetases XabABCD involved in its biosynthesisis. Domains and modules are labeled: adenylation domain (A), peptidyl carrier protein domain (PCP), condensation domain (C), condensation/epimerization domain $(\mathrm{C} / \mathrm{E})$, thioesterase domain $(\mathrm{Te})$.

With alternating $\mathrm{L}$ and $\mathrm{D}$ configuration in the peptide chain, xenoamicins belong to the class of peptides which can form $\beta$ helices in membranes and hence ion channels. The best-studied example is the antibiotic gramicidin $\mathrm{D},{ }^{[34]}$ which is also formed nonribosomally and requires two molecules to span a membrane. Recently, a novel class of ribosomally made alternating $\mathrm{D} / \mathrm{L}$ peptides, namely, proteusins, which has strong cytotoxic activity and polytheonamide as most prominent member, has been identified and its biosynthesis elucidated. ${ }^{[35]}$

In contrast to these $\mathrm{D} / \mathrm{L}$ peptides, 1 most likely cannot form such a $\beta$-helix due to the depsipeptide structure. Nevertheless, the presence of such a large number of hydrophobic amino acids and a $\mathrm{N}$-terminal acyl moiety in $\mathbf{1}$ may indicate an interaction with the membrane. Hence, the bioactivity of xenoamicin A (1) was investigated. Whereas no antibacterial or antifungal activity could be observed in an agar diffusion assay with E. coli DH10B, B. subtilis, M. luteus, P. aeruginosa, $S$. cerevisiae, and $C$. albicans, ${ }^{[36]}$ good activity against Plasmodium falciparum NF $54\left(\mathrm{IC}_{50}\right.$ of $2.35 \mu \mathrm{g} \mathrm{mL}^{-1}$ ) and Trypanosoma brucei rhodesiense STIB900 ( $\mathrm{IC}_{50}$ of $6.41 \mu \mathrm{g} \mathrm{mL}^{-1}$ ) was observed. Much weaker activity against Trypanosoma cruci Tulahuen $\mathrm{C} 4\left(\mathrm{IC}_{50}\right.$ of $\left.30.5 \mu \mathrm{g} \mathrm{mL}^{-1}\right), L$. donnovanni MHOM-ET-67L82 $\left(\mathrm{IC}_{50}\right.$ of $\left.50.1 \mu \mathrm{g} \mathrm{mL}^{-1}\right)$, and mammalian L6 cells ( $\mathrm{IC}_{50}$ of $\left.68.5 \mu \mathrm{g} \mathrm{mL}^{-1}\right)$ indicated a specific target. ${ }^{[37]}$ However, further analysis is needed to identify this target and also to elucidate the natural function of this novel class of compounds in the complex life cycle of Xenorhabdus with its nematode host and insect prey.

\section{Experimental Section}

Xenoamicin A (1): White powder; ${ }^{1} \mathrm{H}$ NMR $\left(600 \mathrm{MHz},\left[\mathrm{D}_{6}\right]\right.$ benzene, $\left.25^{\circ} \mathrm{C}\right)$ : see Table $1 ;{ }^{13} \mathrm{C}$ NMR $\left(150 \mathrm{MHz},\left[\mathrm{D}_{6}\right]\right.$ benzene, $25^{\circ} \mathrm{C}$, see Table 1 ; MS/MS $(70 \mathrm{eV}$ ) and HRMS (MALDI): see Table S1 of the Supporting Information.

Cultivation and extraction: $X$. doucetiae DSM17909 and $X$. mauleonii DSM17908 were always cultivated at $30^{\circ} \mathrm{C}, 200 \mathrm{rpm}$. For inoculation, $1 \%$ overnight preculture and $2 \%$ XAD-16 in LB medium were used. For analytical and preparative HPLC, LB medium was used $(20 \mathrm{~mL}$ and $12 \mathrm{~L}$, respectively). XAD-16 resin beads were collected with a sieve after $72 \mathrm{~h}$, washed with a small amount of water, and extracted with methanol to yield the crude extract $(9.5 \mathrm{~g})$ after evaporation from the large-scale cultivation.

Feeding experiments: The strains DSM17909 and DSM17908 were cultivated at $30^{\circ} \mathrm{C}$ overnight. The cell pellet of the overnight culture was washed once with ISOGRO $-{ }^{13} \mathrm{C}$ medium $(1 \mathrm{~mL})$ and dissolved again in ${ }^{13} \mathrm{C}$ medium $(1 \mathrm{~mL})$. ISOGRO $-{ }^{13} \mathrm{C}$ medium was prepared with ISOGRO ${ }^{13} \mathrm{C}$ powder $(1 \mathrm{~g}), \mathrm{K}_{2} \mathrm{HPO}_{4}\left(1.8 \mathrm{gL}^{-1}\right), \mathrm{KH}_{2} \mathrm{PO}_{4}\left(1.4 \mathrm{gL}^{-1}\right)$, and $\mathrm{CaCl}_{2} \cdot x \mathrm{H}_{2} \mathrm{O}\left(11.1 \mathrm{mgL}^{-1}\right)$ dissolved in $\mathrm{H}_{2} \mathrm{O}(100 \mathrm{~mL})$. The feeding culture in ${ }^{13} \mathrm{C}$ medium $(5 \mathrm{~mL})$ was inoculated with washed overnight culture $(50 \mu \mathrm{L})$. After incubation for $6 \mathrm{~h}$ at $30^{\circ} \mathrm{C}$, a stock solution $(50 \mu \mathrm{L}$, $100 \mathrm{~mm}$ ) of the respective amino acid ( $\beta$-alanine, $\mathrm{L}-\alpha$-threonine, $\mathrm{L}-\alpha$-proline, $\mathrm{L}-\alpha$-isoleucine, $\mathrm{L}-\alpha$-leucine, $\mathrm{L}-\alpha$-alanine, or $\mathrm{L}-\alpha$-valine) was added. Another two additions were carried out at 24 and $48 \mathrm{~h}$. The cultures were extracted with ethyl acetate $(5 \mathrm{~mL})$ after incubation for $72 \mathrm{~h}$. The extract was then prepared for HPLC-MS analyses. ${ }^{[14]}$

Hydrolysis of the extract: The extract was treated with ammonium hydroxide solution (Sigma-Aldrich, 28\%) to open the ring in the depsipeptides ${ }^{[23]}$ Hydrolysis was started with methanol extract solution $(50 \mu \mathrm{L})$ and ammonium hydroxide solution $(500 \mu \mathrm{L})$. After incubation for $2 \mathrm{~h}$ at $40^{\circ} \mathrm{C}$, the reaction mixture was neutralized with $\mathrm{HCl}(3 \mathrm{M})$ solution. After evaporation of the solvent, the sample was then prepared for HPLC-MS analyses. 
HPLC and mass spectrometric analysis of the extract: Analysis of the extracts was carried out by using an Ultimate 3000 LC system from Dionex, coupled to an amaZon X electrospray ionization mass spectrometer from Bruker Daltonics. Peptides were separated on a C18 Column (ACQITY UPLC BEH, $1.7 \mu \mathrm{m}, 2.1 \times 50 \mathrm{~mm}$, flow rate $0.6 \mathrm{~mL} \mathrm{~min}^{-1}$, Waters). Acetonitrile/water containing $0.1 \% \mathrm{HCOOH}$ was used as mobile phase under a linear gradient from $40-50 \%$ acetonitrile over $17.5 \mathrm{~min}$ for the separation. Collision-induced dissociation (CID) was performed on the ion trap in the amaZon $\mathrm{X}$ in positive mode with a scan range of $\mathrm{m} / \mathrm{z}$ 100-1500. ${ }^{[17]}$ HR-MALDI-MS data were obtained with a MALDI LTQ Orbitrap XL from Thermo Fisher Scientific equipped with a $337 \mathrm{~nm}$ nitrogen laser. The extract was diluted in acetonitrile for MALDI-MS analysis. $0.2 \mu \mathrm{L}$ of the extract solution and $0.5 \mu \mathrm{L}$ of a $20 \mathrm{~mm}$ 4-cloro- $\alpha$-cyanocinnamic acid (ClCCA) solution $(70 \%$ acetonitrile) were spotted on the MALDI target and air-dried. The following instrument parameters were used: laser energy, $25 \mu \mathrm{J}$; automatic gain control, on; auto spectrum filter, off; resolution, 100000; plate motion, survey CPS. Mass spectra were internally calibrated with calibration mixture 2 (Applied Biosystems, Sequazyme peptide mass standard kits). The molecular formulas were calculated by using the Qual Browser software V. 2.0.7 (Thermo Fisher Scientific). ${ }^{[18]}$

Advanced Marfey's method: Compound 1 (ca. $0.5 \mathrm{mg}$ ) was hydrolyzed with $\mathrm{HCl}(6 \mathrm{M}, 0.8 \mathrm{~mL})$ in a high-pressure Ace tube at $110^{\circ} \mathrm{C}$ for $16 \mathrm{~h}$. The hydrolysate was evaporated to dryness and resuspended in $\mathrm{H}_{2} \mathrm{O}$ $(100 \mu \mathrm{L})$. To $50 \mu \mathrm{L}$ of this solution $\mathrm{NaHCO}_{3}(10 \mu \mathrm{L}, 1 \mathrm{M})$ and $N_{\alpha}-(5-$ fluoro-2,4-dinitrophenyl)-L-leucinamide or -D-leucinamide (L-FDLA or D-FDLA, $100 \mu \mathrm{L}$ of a $1 \%$ solution in acetone) were added. The reaction vials were covered with aluminum foil and placed in a water bath at $40^{\circ} \mathrm{C}$ for $1 \mathrm{~h}$. Then the reaction mixtures were cooled to room temperature, quenched with $\mathrm{HCl}(10 \mu \mathrm{L}, 1 \mathrm{M})$, and evaporated to dryness. ${ }^{[21,22]}$ The residue was dissolved in methanol $(400 \mu \mathrm{L})$. The analysis of $\mathrm{L}-$ and D-FDLA-modified amino acids was carried out by LC-MS, as mentioned above for the analysis of the extract. Acetonitrile/water containing $0.1 \%$ $\mathrm{HCOOH}$ was used as mobile phase under a linear gradient from $20-60 \%$ acetonitrile over $34 \mathrm{~min}$ for the separation. At same time, the commercial standard amino acids were prepared as reference.

Isolation: For isolation of $\mathbf{1}$, the XAD extract $(9.5 \mathrm{~g})$ of $X$. doucetiae DSM 17909 was dissolved in $50 \mathrm{~mL}$ of DMSO/MeOH/iPrOH (7:2:1). The resulting suspension was centrifuged at $13.3 \times 10^{3} \mathrm{rpm}$ for $4 \mathrm{~min}$ and the pellet was discarded after further analysis. The following preparative HPLC setup was used: $4 \mathrm{~mL}$ per injection by using a Gilson $231 \mathrm{XL}$ sample injector with a sandwich injection method, a Gilson 402 syringe pump, a Gilson 215 liquid handler and two Varian Prep Star SD-1 pumps with a flow rate of $95 \mathrm{~mL} \mathrm{~min}^{-1}$ and an acetonitrile/water gradient from 0-1 $\min 5 \%, 1-16 \min 5-95 \%, 16-19 \min 95 \%$, a Gilson 306 pump for the addition of $10 \% \mathrm{HCOOH}$ buffer with a flow rate of $2.5 \mathrm{~mL} \mathrm{~min}^{-1}$, a Dionex P580 pump for the makeup flow of $1 \mathrm{~mL} \mathrm{~min}^{-1}$ acetonitrile, a Jasco UV 975 recording at $215 \mathrm{~nm}$, an MRA Active Splitter, a Varian 380LC ELSD, and a Luna C18 $10 \mu \mathrm{m} 50 \times 50$ column from Phenomenex (for the chromatogram, see Figure S2 of the Supporting Information). The obtained fractions were freeze-dried. The fractions containing $\mathbf{1}$ were combined and purified in a second step on the same instrument setup, but with a flow rate of $75 \mathrm{~mL} \mathrm{~min}^{-1}$ and an acetonitrile/water gradient from $0-1 \min 45 \%, 1-16 \min 45-65 \%, 16-19 \min 65-95 \%$ and a Luna C18 $5 \mu \mathrm{m} 30 \times 75$ column. The buffer for the second step was changed to $3.33 \%$ trifluoroacetic acid buffer, which was added at a flow rate of $2.5 \mathrm{~mL} \mathrm{~min}^{-1}$ (Figure S3 of the Supporting Information). In a third purification step the selectivity of the chromatographic system was changed through the $\mathrm{pH}$ value. Therefore, an aqueous solution of ammonium acetate $\left(50 \mathrm{gL}^{-1}\right)$ was used as buffer. A flow rate of $75 \mathrm{~mL} \mathrm{~min}^{-1}$ with an acetonitrile/water gradient from 0-1 min 5\%, 1-16 min 5-95\%, $16-19 \min 95 \%$ and a Luna C18 $5 \mu \mathrm{m} 30 \times 75$ column (Figure S4 of the Supporting Information) were used.

NMR spectroscopy: NMR spectra were recorded on a Bruker AVANCE 600 spectrometer operating at a ${ }^{1} \mathrm{H}$ frequency of $600.2 \mathrm{MHz}$ and a ${ }^{13} \mathrm{C}$ frequency of $150.9 \mathrm{MHz}$. The instrument was equipped with a $5 \mathrm{~mm}$ TCI probe head. The final experiments were carried out with a sample of $\mathbf{1}(10 \mathrm{mg})$ dissolved in $\left[\mathrm{D}_{6}\right]$ benzene $(600 \mu \mathrm{L})$ at $293 \mathrm{~K}$. For structure eluci- dation and complete assignment of proton and carbon resonances, 1D ${ }^{1} \mathrm{H}, 1 \mathrm{D}{ }^{13} \mathrm{C}$, DQF-COSY, ROESY (mixing time $150 \mathrm{~ms}$, spinlock field $2 \mathrm{kHz}$ ), multiplicity-edited HSQC, and HMBC spectra were acquired. ${ }^{1} \mathrm{H}$ chemical shifts were referenced to the solvent signals $\left({ }^{1} \mathrm{H}: 7.15 \mathrm{ppm},{ }^{13} \mathrm{C}\right.$ : $128.0 \mathrm{ppm})$.

Two-dimensional homonuclear experiments (DQF-COSY, TOCSY, and ROESY) were performed with a spectral width of $11 \mathrm{ppm}$. Spectra were recorded with 1024 increments in $t_{1}$ and 4096 complex data points in $t_{2}$. For each $t_{1}$ value 4 (DQF-COSY), 8 (TOCSY), or 16 (ROESY) transients were averaged.

For the HSQC spectrum 1024 increments with 3072 complex data points in $t_{2}$ were collected with sweep widths of $8 \mathrm{ppm}$ in the proton and $90 \mathrm{ppm}$ in the carbon dimension. For each $t_{1}$ value four transients were averaged. For the HSQC-TOCSY spectrum 1024 increments with 3072 complex data points in $t_{2}$ were collected with a sweep width of $11 \mathrm{ppm}$ in the proton and $80 \mathrm{ppm}$ in the carbon dimension. For each $t_{1}$ value 32 transients were averaged, and a mixing time of $80 \mathrm{~ms}$ was used for the TOCSY transfer. The HMBC spectrum was acquired with a sweep width of $11 \mathrm{ppm}$ in the proton and $200 \mathrm{ppm}$ in the carbon dimension by using a defocusing delay of $62 \mathrm{~ms}$ (optimized for coupling constants of $8 \mathrm{~Hz}$ ). A total of 32 transients were averaged for each of 1024 increments in $t_{1}$, and 4096 complex points in $t_{2}$.

Identification and verification of the gene cluster: The proposed gene cluster responsible for the biosynthesis of xenoamicins from Xenorhabdus doucetiae DSM17909 (originally strain FRM16) was analyzed by using PKS/NRPS Analysis Web-site (http://nrps.igs.umaryland.edu/ nrps/). ${ }^{[24]}$ To confirm the gene cluster, gene $x a b B$ was disrupted by insertion of a plasmid. Xenorhabdus doucetiae DSM17909 was naturally resistant to ampicillin, which was used to select Xenorhabdus after conjugation. Primer pair 5'-GAACTGGCATGCGGAAATTGAGGCGCAAC and 5'-ACAAGAGAGCTCCCTTCCTGAAGCGGTG were used to amplify parts of gene $x a b B$, which were then digested and cloned into pDS132 vector and transformed into $E$. coli S17 ( $\lambda$ pir). After conjugation, primer pair 5'-GCCCGATATCCTGTCATTG and 5'-ACATGTGGAATTGTGAGCGG was used to verify the insertion of the plasmid in the mutants. The detailed method was used by us before. ${ }^{[38]}$ The extract from the mutant was prepared as for the wild type, except for chloramphenicol in the LB medium for the mutant.

\section{Acknowledgements}

Work in the Bode lab was supported by the excellence initiative of the Hessian Ministry of Science and Art via the LOEWE research focus "Insect Biotechnology" and a European Research Council starting grant under grant agreement no. 311477. The authors are grateful to Genoscope (Evry, France) for access to the $X$. doucetiae genome prior to publication.

[1] H. B. Bode, Angew. Chem. 2009, 121, 6512-6514; Angew. Chem. Int Ed. 2009, 48, 6394-6396.

[2] C. Corre, G. Challis, Chem. Biol. 2007, 14, 7.

[3] L. Song, F. Barona-Gomez, C. Corre, J. Am. Chem. Soc. 2006, 128, $14754-14755$.

[4] H. B. Bode, Curr. Opin. Chem. Biol. 2009, 13, 224-230.

[5] H. Goodrich-Blair, Curr. Opin. Microbiol. 2007, 10, 225-230.

[6] E. E. Herbert, H. Goodrich-Blair, Nat. Rev. Microbiol. 2007, 5, 634646.

[7] D. Ji, Y. Yi, G. H. Kang, Y. H. Choi, P. Kim, N. I. Baek, Y. Kim, FEMS Microbiol Lett. 2004, 239, 241-248.

[8] J. Crawford, C. Portmann, X. Zhang, Proc. Natl. Acad. Sci. USA 2012, 109, 10821-10826.

[9] B. McInerney, W. Taylor, M. Lacey, R. Akhurst, R. Gregson, J. Nat. Prod. 1991, 54, 785-795.

[10] T. Hjelmgaard, M. Givskov, J. Nielsen, Org. Biomol. Chem. 2007, 5, 344-348. 
[11] A. O. Brachmann, S. Forst, G. M. Furgani, A. Fodor, H. B. Bode, J. Nat. Prod. 2006, 69, 1830-1832.

[12] J. Li, G. Chen, J. M. Webster, Can. J. Microbiol. 1997, 43, 770-773.

[13] S. W. Fuchs, A. Proschak, T. W. Jaskolla, M. Karas, H. B. Bode, Org. Biomol. Chem. 2011, 9, 3130-3132.

[14] H. B. Bode, D. Reimer, S. W. Fuchs, F. Kirchner, C. Dauth, C. Kegler, W. Lorenzen, A. O. Brachmann, P. Grün, Chem. Eur. J. 2012, 18, 2342-2348.

[15] G. Lang, T. Kalvelage, A. Peters, J. Wiese, J. F. Imhoff, J. Nat. Prod. 2008, 71, 1074-1077.

[16] Q. Zhou, A. Dowling, H. Heide, J. Wöhnert, U. Brandt, J. Baum, R. ffrench-Constant, H. B. Bode, J. Nat. Prod. 2012, 75, 1717-1722.

[17] F. Grundmann, V. Dill, A. Dowling, A. Thanwisai, E. Bode, N. Chantratita, R. ffrench-Constant, H. B. Bode, Beilstein J. Org. Chem. 2012, 8, 749-752.

[18] S. W. Fuchs, C. C. Sachs, C. Kegler, F. I. Nollmann, M. Karas, H. B. Bode, Anal. Chem. 2012, 84, 6948-6955.

[19] C. R. Mitchell, Y. Bao, N. J. Benz, S. Zhang, J. Chromatogr. B 2009, 877, 4133-4139.

[20] M. Schubert, D. Labudde, H. Oschkinat, P. Schmieder, J. Biomol. NMR 2002, 24, 149-154.

[21] K. Fujii, Y. Ikai, H. Oka, M. Suzuki, K. Harada, Anal. Chem. 1997, 69, 5146-5151.

[22] K. Fujii, T. Shimoya, Y. Ikai, H. Oka, K. Harada, Tetrahedron Lett. 1998, 39, 2579-2582.

[23] I. Vallet-Gely, A. Novikov, L. Augusto, P. Liehl, G. Bolbach, M. Pechy-Tarr, P. Cosson, C. Keel, M. Caroff, B. Lemaitre, Appl. Environ. Microbiol. 2010, 76, 910-921.

[24] B. O. Bachmann, J. Ravel, Meth. Enzymol. 2009, 458, 181-217.

[25] M. H. Medema, K. Blin, P. Cimermancic, V. de Jager, P. Zakrzewski, M. A. Fischbach, T. Weber, E. Takano, R. Breitling, Nucleic Acids Res. 2011, 39, W339-W346.
[26] K. Blin, M. H. Medema, D. Kazempour, M. A. Fischbach, R. Brei tling, E. Takano, T. Weber, Nucleic Acids Res. 2013, 41, W204W212.

[27] S. F. Altschul, T. L. Madden, A. A. Schäffer, J. Zhang, Z. Zhang, W. Miller, D. J. Lipman, Nucleic Acids Res. 1997, 25, 3389-3402.

[28] T. Weber, K. J. Laiple, E. K. Pross, A. Textor, S. Grond, K. Welzel, S. Pelzer, A. Vente, W. Wohlleben, Chem. Biol. 2008, 15, 175-188.

[29] M. Rottig, M. H. Medema, K. Blin, T. Weber, C. Rausch, O. Kohlbacher, Nucleic Acids Res. 2011, 39, W362-W367.

[30] C. Rausch, T. Weber, O. Kohlbacher, W. Wohlleben, D. H. Huson, Nucleic Acids Res. 2005, 33, 5799-5808.

[31] T. Stachelhaus, H. D. Mootz, M. A. Marahiel, Chem. Biol. 1999, 6 , 493-505.

[32] D. Konz, M. A. Marahiel, Chem. Biol. 1999, 6, R39-48.

[33] C. J. Balibar, F. H. Vaillancourt, C. T. Walsh, Chem. Biol. 2005, 12, 1189-1200.

[34] B. M. Burkhart, R. M. Gassman, D. A. Langs, W. A. Pangborn, W. L. Duax, V. Pletnev, Biopolymers 1999, 51, 129-144.

[35] M. F. Freeman, C. Gurgui, M. J. Helf, B. I. Morinaka, A. R. Uria, N. J. Oldham, H. Sahl, S. Matsunaga, J. Piel, Science 2012, 338, $387-$ 390.

[36] D. Velasco, M. M. del Tomas, M. Cartelle, A. Beceiro, A. Perez, F. Molina, R. Moure, R. Villanueva, G. Bou, J. Antimicrob. Chemother. 2005, 55, 379-382.

[37] F. I. Nollmann, F. I. Nollmann, A. Dowling, M. Kaiser, K. Deckmann, S. Grösch, R. ffrench-Constant, H. B. Bode, H. B. Bode, Beilstein J. Org. Chem. 2012, 8, 528-533.

[38] A. O. Brachmann, S. A. Joyce, H. Jenke-Kodama, G. Schwär, D. J. Clarke, H. B. Bode, ChemBioChem 2007, 8, 1721-1728.

Received: June 27, 2013 Published online: $\mathbf{\square}$ al, 0000 
Large and alternating: Xenoamicins, novel tridecadepsipeptides with alternating $\mathrm{D}$ - and L-amino acid residues, were isolated from Xenorhabdus sp (see scheme). Their structures were elucidated by detailed NMR experiments, the advanced Marfey's method, and feeding experiments followed by $\mathrm{MS}^{n}$ studies. Additionally, identification of the the biosynthesis gene cluster indicated promiscuity of specific domains leading to the various derivatives identified.

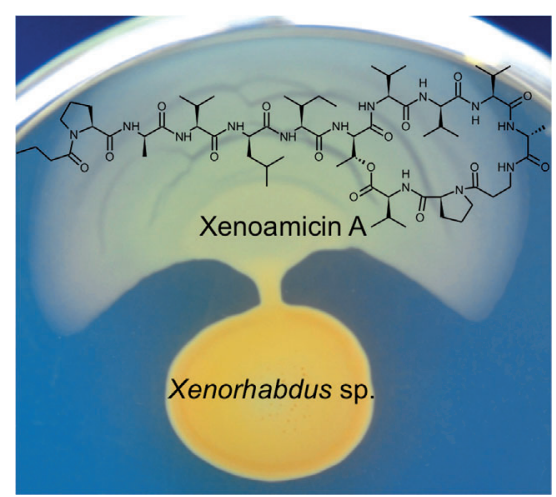

Natural Products

Q. Zhou, F. Grundmann, M. Kaiser, M. Schiell, S. Gaudriault, A. Batzer,

M. Kurz, H. B. Bode*

IIII-III

Structure and Biosynthesis of Xenoamicins from Entomopathogenic Xenorhabdus 